\title{
Three-dimensional viscous flow over an unsteady permeable stretching/shrinking sheet
}

\begin{abstract}
In this study, a numerical investigation on the unsteady three-dimensional boundary layer flow of a viscous fluid past a permeable stretching/shrinking sheet is considered. Similarity transformation is employed to reduce the governing system of nonlinear partial differential equations into the ordinary (similarity) differential equations. These equations are then solved numerically by using a shooting method. Both stretching and shrinking cases are considered. Effects of the unsteadiness parameter, stretching/shrinking parameter, mass suction parameter and ratio of the surface velocity gradients along the vertical $y$ - and horizontal $x$ - directions are presented and discussed in detail. The numerical results show that for the shrinking case, the skin friction coefficient and the velocity boundary layer thickness increase with increasing unsteadiness parameter, while the skin friction coefficient decreases and the velocity boundary layer thickness increases with increasing ratio of the surface velocity gradients. The results also show that dual solutions exist for both cases of stretching and shrinking sheet.
\end{abstract}

Keyword: Boundary layer; Numerical solutions; Stretching/shrinking sheet; Threedimensional flow; Unsteady flow 